\title{
Helicobacter pylori infection, H19 and LINC00152 expression in serum and risk of gastric cancer in a Chinese population
}

Tian Yang ${ }^{\mathrm{a}^{*}}$, Hongmei Zeng ${ }^{\mathrm{b}^{*}} \uparrow$, Wanqing Chen ${ }^{\mathrm{b}}$, Rongshou Zheng ${ }^{\mathrm{b}}$, Yang Zhang ${ }^{\mathrm{a}}$, Zhexuan $\mathrm{Li}^{\mathrm{a}}$, Jun $\mathrm{Qi}^{\mathrm{c}}$, Minjie Wang ${ }^{\mathrm{c}}$, Tianhui Chen ${ }^{\mathrm{d}}$, Jianlin Lou ${ }^{\mathrm{d}}$, Lingeng Lu ${ }^{\mathrm{e}}$, Tong Zhou $^{\mathrm{a}}$, Shuyang Dai ${ }^{\mathrm{f}}$, Meng Cai ${ }^{\mathrm{f}}$, Weicheng You $^{\mathrm{a}}$, Kaifeng $\operatorname{Pan}^{\mathrm{a}}{ }_{\dagger}$

\section{Author's Affiliations:}

a. Key Laboratory of Carcinogenesis and Translational Research (Ministry of Education/Beijing), Department of Cancer Epidemiology, Peking University School of Oncology, Peking University Cancer Hospital \& Institute, Beijing, China.

b. National Office for Cancer Prevention and Control, National Cancer Center/Cancer Hospital, Chinese Academy of Medical Sciences and Peking Union Medical College, Beijing, China.

c. Department of Clinical Laboratory, National Cancer Center/Cancer Hospital, Chinese Academy of Medical Sciences and Peking Union Medical College, Beijing, China.

d. Institute of Occupational Diseases, Zhejiang Academy of Medical Sciences, Hangzhou, China.

e. Department of Chronic Disease Epidemiology, Yale School of Public Health, School of Medicine, Yale Cancer Center, Yale University, New Haven, USA

f. State Key Laboratory of Molecular Oncology, National Cancer Center/Cancer Hospital, Chinese Academy of Medical Sciences and Peking Union Medical College, Beijing, China.

*The first two authors contributed equally to the study.

$\uparrow$ Corresponding author: Hongmei Zeng, National Office for Cancer Prevention and Control, National Cancer Center/Cancer Hospital, Chinese Academy of Medical Sciences and Peking Union Medical College, Beijing, China. Fax: +86 1067718227. Email: hongmeizeng2011@163.com.

$†$ Corresponding author: Pan Kaifeng: Key Laboratory of Carcinogenesis and 
Translational Research (Ministry of Education/Beijing), Department of Cancer

Epidemiology, Peking University School of Oncology, Peking University Cancer

Hospital \& Institute, Beijing, China. Fax: +86 10 67718227. Email:

pankaifeng2002@yahoo.com

Running title: lncRNAs, $H$. pylori and gastric cancer 


\begin{abstract}
Gastric cancer (GC) is a consequence of multifactorial and multistep processes. Helicobacter pylori (H. pylori) infection plays a crucial role in gastric carcinogenesis. Long non-coding RNAs (lncRNAs) have shown great potential as powerful cancer biomarkers. To investigate the possible roles of lncRNAs and $H$. pylori infection in GC development, we measured expression levels of three lncRNAs (H19, LINC00152, uc001lsz) in serum from a total of 285 Chinese participants using reverse transcription-quantitative polymerase chain reaction. We found significant associations between high expression of both H19 and LINC00152 in serum and increased risk of GC; the adjusted OR for H19 was 2.17 (95\% CI: 1.21-3.88), and for LINC00152 was 2.09 (95\% CI: 1.18-3.70). Further analyses indicated an elevated risk of GC in subjects with both high H19 expression and H. pylori infection (OR: 13.75, 95\% CI: 4.75-39.84). Significant joint effect between LINC00152 and H. pylori infection on risk of GC was also found (OR: 17.49, 95\% CI: 4.78-63.92). Serum H19 and LINC00152 may serve as potential biomarkers for diagnosis of GC, particularly for those with $H$. pylori infection.
\end{abstract}

Key words: gastric cancer, long non-coding RNA, serum, Helicobacter pylori, biomarkers

Abbreviations: lncRNA, long non-coding RNA; gastric cancer, GC; RT-qPCR, Reverse Transcription-quantitative Polymerase Chain Reaction; OR, odds ratio; CI, confidence interval; SV, stability value; AUC: area under the ROC curve 


\section{Introduction}

Gastric cancer (GC) is the fifth most common cancer and the third leading cause of cancer death for both men and women worldwide [1]. In 2015, 679,000 new cases and 498,000 new deaths due to GC were estimated in China [2]. The most common risk factor of GC is Helicobacter pylori (H. pylori) infection, accounting for more than $70 \%$ of all cases [3]. Certain types of $H$. pylori have higher risks than others such as cytotoxin-associated antigen $\mathrm{A}(\mathrm{CagA})$ and vacuolating toxin (VacA) positive subtypes [4-6]. Interactions between subtypes of pathogen and host, environmental factors, and genetic factors, may be involved in the etiology of GC.

Long non-coding RNA (lncRNA) is generally defined as an RNA molecule with a transcript length of more than $200 \mathrm{nts}$ that lacks coding potential [7]. LncRNAs play critical regulatory roles in cell signaling and regulation [7, 8]. Dysregulation of lncRNAs are closely associated with cancers of the colon, breast, lung and stomach [9-12]. LncRNAs may play oncogenic and tumor suppressor roles in the development of GC $[12,13]$. Circulating lncRNAs can stably exist in serum, thereby may function as promising biomarkers for early detection of GC [14].

Previous studies have demonstrated that lncRNAs, such as H19 and LINC00152 are remarkably elevated in blood and tumor tissues of GC patients [15]. While, uc001lsz expression levels decreased in GC cell lines and tumor tissues [16]. However, the potential joint effect between H.pylori infection and lncRNAs on risk of GC was unknown. Therefore, we aimed at investigating their associations with GC using a larger sample size. We also measured those lncRNAs in GC patients before and after surgical operation, respectively. We further explored potential diagnostic value of lncRNAs and $H$. pylori infection status on GC.

\section{Material and Methods}

\subsection{Study subjects}

Overall 285 subjects were included in our study. A total of 133 pre-operative serum 
samples were collected from pathologically verified GC patients. Among them, 94 cases were diagnosed at the Cancer Hospital, Chinese Academy of Medical Sciences between December 2014 and April 2015; the other 39 were diagnosed by an endoscopic screening program conducted in Sheyang County, Jiangsu Province, China during the same period. Of the $133 \mathrm{GC}$ cases, the post-operative serum samples of 19 patients who did not receive either chemo- or radiotherapy were also collected three months later after surgery. GCs with specific pathological stage were classified into early and advanced stage by WHO standard [17]. One hundred fifty two control samples pathologically diagnosed as normal or superficial gastritis were also collected

from the endoscopic screening program in Sheyang County, Jiangsu Province of China. Information on age, gender, smoking history and drinking for each participant was obtained through a standard structured questionnaire. Ethical approval from the Institutional Review Boards of Cancer Hospital, Chinese Academy of Medical Sciences was obtained and all participants provided written informed consent. The human gastric cell line BGC-823 was gifted from the Department of Etiology, School of Oncology, Peking University Cancer Hospital \& Institute.

\subsection{The evaluation of internal controls for the qualification of serum IncRNAs}

There has been no consensus on stable and suitable internal controls for non-coding RNAs in human serum samples $[18,19]$. Therefore, we aimed to evaluate potential stable internal controls for the qualification of the serum lncRNAs in our study. Six candidate reference housekeeping genes (GAPDH, $\beta$-actin, HPRT1, 18S rRNA, B2M and RPL29) were evaluated in 24 serum samples, including 12 GC patients and 12 healthy controls for a reliable internal control. NormFinder V12 software, accounting for both intra- and inter-group variations to evaluate the stability of each single reference gene, was used in our study [20]. The top four genes with smallest stability values (SV) were further investigated for their stability through repeated freezing and thawing cycles. Four subjects' specimens were randomly selected and each sample was divided into five portions. These sample portions were subjected to freezing (at 
$-20^{\circ} \mathrm{C}$ ) and thawing (at room temperature) $0,1,3,6$ and 9 times before the extraction of RNA.

\subsection{Blood sample processing, RNA extraction and Reverse transcription}

Up to $5 \mathrm{ml}$ of blood was collected from each participant between December of 2014 and April of 2015. Blood samples were allowed to stand for 30-40 min and serum separation was accomplished by centrifugation at $965 \mathrm{~g}$ for $15 \mathrm{~min}$. The supernatant serum was recovered and stored at $-80^{\circ} \mathrm{C}$ until the detection. Total RNA was isolated from $100 \mu \mathrm{L}$ of the cultured cells and $400 \mu \mathrm{L}$ of serum using a mirVana PARIS KIT (Ambion, Austin, TX, USA). Complementary DNA was generated using the GoScript Reverse Transcription (RT) System (Promega, Madison, WI). RT reaction and no-template control were run at the same time for quality control.

\subsection{Quantitative real-time PCR}

Quantitative polymerase chain reaction (qPCR) was performed using the GoTaq qPCR Master Mix (Promega, Madison, WI) on an Applied Biosystems 7500 FAST Real-time PCR system (Applied Biosystems). The primers used in this study and their denaturing temperatures are presented in supplementary material (S1). All the primers were synthesized by Sangon Biotech (Shanghai, China). The expression levels of the candidate genes were measured in triplicate. Relative quantification of target gene

expression was performed with $2^{-\Delta \Delta \mathrm{CT}}$ method by dividing the lncRNA/ $\beta$-actin ratio of a tested sample by the lncRNA / $\beta$-actin ratio of a reference sample. The $\beta$-actin gene was used as an internal reference gene to normalize RNA input. The RNA extracted from BGC-823 cell line with fixed expression levels of H19 and LINC00152 acted as the reference sample on every PCR detection plate, to which all the serum samples were compared.

\subsection{H. pylori multiplex serology}

Sero-status of antibodies to four H. pylori specific antigens (CagA, VacA, UreA and UreB) was determined by Western Blot using Typing Detection Kit for Antibody to $H$. 
pylori (Shenzhen Blot Biotech Co., Ltd, Shenzhen, China). It allows the identification of specific antibody responses against distinct $H$. pylori antigens of CagA, VacA, UreA and UreB. Highly purified $H$. pylori antigens were individually immobilized on membrane strips according to the manufacturer's instructions. The test strips were incubated with the diluted serum sample (dilution 1:100 with wash buffer A) for 30 min at room temperature with gentle shaking. After washing, the strips were incubated with anti-human immune globulin antibodies ( $\mathrm{IgG}$, dilution 1:100) for $30 \mathrm{~min}$ at room temperature. Unbound conjugate antibodies were removed by washing three times. Bound antibodies were detected through a staining reaction that would reveal a dark band on the strip in the corresponding antigen lane, and the subject would be specific H. pylori seropositive. H. pylori sero-positivity was defined as any of the CagA, VacA, UreA or UreB being seropositive. Subjects with CagA and/or VacA positivity were classified into Type I $H$. pylori seropositive. Those with only UreA and/or UreB positivity were classified into Type II $H$. pylori seropositive.

\subsection{Statistical analysis}

Pearson's $\chi^{2}$ test was used to compare the difference between cases and controls on age, sex, smoking, drinking and $H$. pylori infection status. The Wilcoxon rank test was used to evaluate difference between the lncRNAs levels in pre- and postoperative pared serum samples. For data analysis, the lncRNAs expression levels were analyzed as categorical variables with 2 ordinal levels, low and high. We dichotomized the normalized expression data using the median in control subjects as the cutoff to define H19/LIN00152 expression as high expression (expression level>median in control group) and low expression (expression level<=median in control group). Odds ratios (ORs) and 95\% confidence intervals (CIs) were calculated for the association of lncRNAs expression levels with $H$. pylori infection status and with the risk of GC, as well as for lncRNA-H. pylori joint effect, using unconditional logistic regression. All analyses were adjusted for potential confounders. For lncRNA- $H$. pylori joint effect analysis, we generated a new variable combined with two factors; ORs and 95\% CIs were estimated by adding the new variable into the unconditional logistic model. 
Multiplicative lncRNA-H. pylori interactions were measured by including main effect variables and their product terms in the logistic regression model, using likelihood ratio tests. Multivariate classification models were constructed to determine the best panel for GC prediction using logistic regression. Receiving operating characteristic (ROC) curves were established to evaluate the diagnostic value of each panel. SPSS 19.0 software (SPSS, NY) was used for data analysis. A two-sided $P$ value $<0.05$ was considered statistically significant.

\section{Results}

\subsection{Basic characteristics of study subjects}

A total of 285 subjects were enrolled in this study, including 133 GC cases and 152 controls. Detailed information on the basic characteristics of the study subjects is presented in Table 1. There were no significant differences in age, sex and smoking between the GC patients and the controls. However, the distributions of both drinking and $H$. pylori infection status were significantly different between the two groups $(P<0.001)$. The proportions of drinking and $H$. pylori infection status were significantly higher in GC patients compared to the controls. Especially, the proportions of CagA+ and VacA + H.pylori infection were significantly higher in GC cases than in controls. Among all patients who had detailed pathological information on stage, 40 cases $(30.1 \%)$ were in early stage and $57(42.8 \%)$ belonged to advanced stage, and $75.2 \%$ of cases occurred at non-cardiac sites of the stomach.

\subsection{Evaluation of potential internal controls for the qualification of serum} IncRNAs

In order to identify the most stable reference genes, we examined the levels of GAPDH, $\beta$-actin, HPRT1, 18S rRNA, B2M and RPL29 in 24 serum samples. The mean CT value and standard deviation (SD) of each tested gene is shown in supplementary material (S2). $\beta$-actin was ranked as the most suitable reference gene with SV of 0.063 . Further stability analysis showed that $\beta$-actin levels did not change significantly after repeated cycles of freezing/thawing (supplementary material: S3). 
Therefore, we used $\beta$-actin as the internal control for our study.

\subsection{Serum IncRNAs expression in cases and controls}

Three candidate lncRNAs (H19, LINC00152 and uc001lsz) were measured to test their detectability and stability in serum. We found that both H19 and LINC00152 were stably detectable in serum. However, uc001lsz could not be detected in human serum among all subjects. Therefore, we only compared the levels of H19 and LINC00152 between GC patients and controls in the 285 study subjects. We found by multivariate analyses that serum expression levels for both H19 and LINC00152 were significantly higher in cases than in controls, reaching an OR of 2.17 (95\% CI: 1.21-3.88) for $\mathrm{H} 19$ and 2.09 (95\% CI: 1.18-3.70) for LINC00152. In addition, subjects with high expression levels for both H19 and LINC00152 had significantly elevated risk of GC, compared to those with low expression levels for both H19 and LINC00152, with adjusted OR of 4.13 (95\% CI: 1.58-10.79) (Table 2).

\subsection{Stratified analysis by $H$. pylori infection status}

Stratified analyses by $H$. pylori infection status are presented in Table 3 . We found significantly elevated risk for subjects with $H$. pylori infection and high expression of H19 (2.27, 95\% CI: 1.19-4.33). For LINC00152, we also found significantly elevated risk for subjects with $H$. pylori infection and high expression of LINC00152 (2.04, 95\% CI: $1.09-3.82)$.

\subsection{Analysis of joint effects between IncRNAs and $\boldsymbol{H}$. pylori infection}

We also evaluated the joint effects of lncRNAs and H. pylori infection on GC (Table 4). For H19, we found 6-fold elevated GC risk for subjects with low expression of H19 and with $H$. pylori infection (5.75, 95\% CI: 1.97-16.66), but 14-fold elevated risk for those with high expression of $\mathrm{H} 19$ and $H$. pylori infection $(13.75,95 \% \mathrm{CI}$ : 4.75-39.84). For LINC00152, we found 9-fold elevated risk for subjects with low expression of LINC00152 and with H. pylori infection (8.55, 95\% CI: 2.38-30.78), but 17-fold elevated risk for those with high expression of LINC00152 and $\mathrm{H}$. pylori 
infection (17.49, 95\% CI: 4.78-63.92). However, no statistically significant multiplicative or additive interaction between H19/LINC00152 and H.pylori infection was found.

\subsection{Stratified analysis by type of $H$. pylori infection}

We made further analysis on type of $H$. pylori infection and lncRNAs expression (Table 5). For H19, we found that only in subjects with Type I H. pylori infection, there was a significant association between $\mathrm{H} 19$ expression and $\mathrm{GC}$ risk $(4.23,95 \% \mathrm{CI}$ : 1.80-9.94). Similarly, only in subjects with Type I H.pylori infection, significant association between LINC00152 expression and GC risk was found (2.86, 95\%CI: 1.22-6.69).

\subsection{Comparison of IncRNA levels between pre- and postoperative paired serum samples}

We compared the levels of H19 and LINC00152 in 19 pre- and postoperative paired serum samples with GC (Fig. 1.). We found that H19 levels were significantly lower in postoperative cases compared to preoperative cases $(P=0.010)$. As for LINC00152, there was no significant difference between the two cases.

\subsection{Potential diagnostic value of IncRNAs and $H$. pylori infection status}

To evaluate the diagnostic value of serum lncRNAs and $H$. pylori infection status on

GC, prediction models were constructed for classification between GC and controls. ROC curves on lncRNAs and $H$. pylori infection were further described. The results revealed that a panel of three factors (H19, LINC00152 and H. pylori infection status) yielded the largest area under the ROC curves (AUCs). The sensitivity, specificity and AUC for the panel were $63.9 \%, 76.3 \%$ and 0.75 (95\% CI: 0.70-0.81), respectively (Fig. 2.).

\section{Discussion}

We investigated three lncRNAs (H19, LINC00152, uc001lsz) in serum samples from 
285 Chinese subjects and found that H19 and LINC00152 were associated with increased risk of GC in a Chinese population. To the best of our knowledge, this is the first study investigating the association between serum lncRNA expression and $H$. pylori infection status with GC risk. We found significant joint effects between H19/LINC00152 expression and H. pylori infection on risk of GC. Our findings of the associations of H19/LINC00152 with GC risk in a Chinese population were consistent with previous reports $[21,22]$. A panel of three indicators (H19, LINC00152 and $H$. pylori infection status) could distinguish GCs from controls.

LncRNAs have been shown to play important regulatory roles and involved in many aspects of cancer biology including chromatin structure, gene expression and translational control [7, 8]. The $H 19$ gene, located in an imprinted region of chromosome 11 near the insulin-like growth factor 2 (IGF2), is only expressed in the maternally-inherited chromosome. $H 19$ has been shown to function as an oncogene in previous studies [23, 24]. Consistent with previous reports [14, 22], our study found that H19 was significantly higher in the serum of GC patients compared to controls, suggesting that H19 is an independent risk factor for GC. H19 has been aberrantly expressed among different stages of GC tissues, juices and normal controls, as previously reported $[12,25,26]$, suggesting H19 may play an important role in the development of GC. For instance, Li et al. found that H19 in GC is mediated by the direct up-regulation of ISM1 and the indirect suppression of CALN1 expression via miR-675 [27]. Zhuang et al showed that H19-produced microRNA-675 (miR-675) regulates GC cell proliferation by targeting RUNX1. Moreover, H19 can be induced by the oncogene c-Myc and plays a key role in the development and progression of GC by regulating cell proliferation [28].

LINC00152 gene, a new long intergenic noncoding RNA, is located at chromosome 2p11.2. Previous studies have shown that LINC00152 is a GC- related lncRNA, and its expression levels in GC were significantly higher compared to non-tumorous tissues $[15,29,30]$. The aberrant expression of LINC00152 between GC and controls 
was also shown in gastric juices [15]. LINC00152 may promote cell proliferation in vitro and tumor growth in vivo, serving an oncogenic function [30-32], although the detailed mechanism of LINC00152 remains unknown. Zhao et al found that LINC00152 was involved in cell cycle and invasion in GC [31]. LINC00152 was also shown to promote proliferation in GC through the EGFR-dependent pathway [30]. Our study adds to further evidence that LINC00152 may function in gastric carcinogenesis. It is reported that uc001lsz was down-regulated in GC tissues compared to non-tumorous tissues, and so was in many GC cell lines [16]. However, no studies have reported the levels of uc0011sz in human serum. To the best of our knowledge, our study was the first one which showed that uc001lsz cannot be detected in serum samples. It is possible that uc001lsz may be too low to be detected in human serum.

The etiology of GC is caused by multiple genetic dysregulation in combination with various environmental factors and bacterial virulence factors [33-35]. H. pylori can induce an inflammatory response in the gastric mucosa and stimulate host innate and adaptive immune responses $[34,36]$. Host response to $H$. pylori may influence the outcome of gastric lesions. The expression pattern of serum lncRNAs and $\mathrm{H}$. pylori infection on risk of GC has rarely been reported. Yang et al. found that two differentially expressed IncRNAs (XLOC_004122, XLOC_014388) might be involved in the immune response against $H$. pylori infection, and LINC00152 was shown to be up-regulated in $H$. pylori-positive gastric tissues [37]. Yao et al. found that serum Lnc-SGK1 expression in combination with $H$. pylori infection in T cells was associated with poor prognosis of GC patients [38]. The joint effects between H19/LINC00152 and H. pylori infection on risk of GC found by our study suggest that subjects with H. pylori infection, especially those with $\mathrm{CagA}+$ or $\mathrm{VacA}+$ sero-positivity and high expression of H19/LINC00152 may be more vulnerable to GC.

We found serum H19 was significantly down expressed after surgery, consistent with 
previous reports [14], suggesting H19 may possibly be released from primary GC in two forms, free and extracellular vesicles. Exosomes are bilayer membrane nano particles, which can protect RNAs, including lncRNAs from degradation in serum [39]. Li et al. reported no significant differences of LINC00152 levels between serum and exosomes, indicating that some lncRNAs detected in serum are mainly in exosomes [21].

Conventional serological markers of CEA and CA19-9 on GC have low specificity and sensitivity on GC diagnosis [18, 40]. Circulating RNAs in serum have been an emerging field for non-invasive diagnostic applications. Studies found that many serum lncRNAs might be used as biomarkers of cancer development [14, 18]. We found the panel with H19, LINC00152 and H. pylori infection status could distinguish serum samples of GC from controls, suggesting that this panel might serve as a biomarker for GC diagnosis. It also raises the possibility that the panel of lncRNAs and $H$. pylori infection status may be useful as a preliminary screening method for people at high risk of GC.

Our study has several strengths. First, we systematically assessed the associations between the levels of $\mathrm{H} 19$ and LINC00152 in serum and the risk of GC in pathologically diagnosed subjects from a total of 285 subjects of China. It is the first study to identify a joint effect between levels of lncRNAs in serum and $H$. pylori infection status on the risk of GC. Our study also has some limitations. We did not find statistically significant interaction between H19/LINC00152 and H. pylori infection. One of the most likely reasons is that the sample size of the study was insufficient to provide enough power for stratification and interaction analysis. Thus, our studies need to be validated in independent studies with larger sample sizes and the functional relevance of the lncRNAs needs to be further clarified.

Taken together, this is the first study investigating the joint effect of $H$. pylori infection and serum lncRNA expression on risk of GC. We found in 285 Chinese 
subjects that serum H19 may potentially be useful for diagnosis of GC and for monitoring after GC operation. Our findings also suggest that expression profiles of lncRNAs and H.pylori infection status could be used as diagnostic markers of GC. Our study provides valuable insights into the bacteria and the lncRNA expression involved in gastric carcinogenesis. 


\section{Conflict of interest}

The authors declare no conflict of interest.

\section{Authorship contribution}

Hongmei Zeng, Wanqing Chen, Weicheng You and Kaifeng Pan were involved in the conception and design of the study. Hongmei Zeng, Tian Yang, Jun Qi, Minjie Wang, Tong Zhou, Shuyang Dai and Meng Cai were involved in the acquisition of data. Tian Yang, Hongmei Zeng and Rongshou Zheng were involved in the data analysis. Tian Yang, Hongmei Zeng, Lingeng Lu, Tianhui Chen, Xuanzhe Li, Yang Zhang and Jianwei Lou were involved in the interpretation of the data. Tian Yang, Hongmei Zeng and Kaifeng Pan drafted the manuscript. Weicheng You, Kaifeng Pan, Jianlin Lou, Lingeng Lu and Hongmei Zeng revised the article. The final manuscript was approved by all authors.

\section{Acknowledgments}

We would like to thank Shanru Gu, Shuguang Dai and Chunyun Dai from Center for

Disease Control and Prevention of Sheyang County for assistance in sample collection and management.

\section{Funding Sources}

This study was supported by Open Fund of Key Laboratory of Carcinogenesis and Translational Research (Ministry of Education/Beijing) [grant number: 2014Open-1], National Science Foundation of China [grant number: 81302504] and Nonprofit Industry Research Fund of National Health and Family Planning Commission of China [grant number: 201502001].

\section{References}

[1] J. Ferlay, I. Soerjomataram, R. Dikshit, S. Eser, C. Mathers, M. Rebelo, D.M. Parkin, D. Forman, F. Bray, Cancer incidence and mortality worldwide: sources, methods and major patterns in GLOBOCAN 2012, International journal of cancer 136(5) (2015) E359-86.

[2] W. Chen, R. Zheng, P.D. Baade, S. Zhang, H. Zeng, F. Bray, A. Jemal, X.Q. Yu, J. He, Cancer statistics in China, 2015, CA: a cancer journal for clinicians 66(2) (2016) 115-32.

[3] M. Plummer, S. Franceschi, J. Vignat, D. Forman, C. de Martel, Global burden of gastric cancer 
attributable to Helicobacter pylori, International journal of cancer 136(2) (2015) 487-90.

[4] J.C. Atherton, T.L. Cover, R.J. Twells, M.R. Morales, C.J. Hawkey, M.J. Blaser, Simple and accurate PCR-based system for typing vacuolating cytotoxin alleles of Helicobacter pylori, Journal of clinical microbiology 37(9) (1999) 2979-82.

[5] T.L. Cover, S.R. Blanke, Helicobacter pylori VacA, a paradigm for toxin multifunctionality, Nature reviews. Microbiology 3(4) (2005) 320-32.

[6] A. Oldani, M. Cormont, V. Hofman, V. Chiozzi, O. Oregioni, A. Canonici, A. Sciullo, P. Sommi, A. Fabbri, V. Ricci, P. Boquet, Helicobacter pylori counteracts the apoptotic action of its VacA toxin by injecting the CagA protein into gastric epithelial cells, PLoS pathogens 5(10) (2009) e1000603.

[7] T.R. Mercer, M.E. Dinger, J.S. Mattick, Long non-coding RNAs: insights into functions, Nature reviews. Genetics 10(3) (2009) 155-9.

[8] X. Li, Z. Wu, X. Fu, W. Han, IncRNAs: insights into their function and mechanics in underlying disorders, Mutation research. Reviews in mutation research 762 (2014) 1-21.

[9] K. Taniue, A. Kurimoto, H. Sugimasa, E. Nasu, Y. Takeda, K. Iwasaki, T. Nagashima, M. Okada-Hatakeyama, M. Oyama, H. Kozuka-Hata, M. Hiyoshi, J. Kitayama, L. Negishi, Y. Kawasaki, T. Akiyama, Long noncoding RNA UPAT promotes colon tumorigenesis by inhibiting degradation of UHRF1, Proceedings of the National Academy of Sciences of the United States of America 113(5) (2016) 1273-8.

[10] Y. Shen, D. Katsaros, L.W. Loo, B.Y. Hernandez, C. Chong, E.M. Canuto, N. Biglia, L. Lu, H. Risch, W.M. Chu, H. Yu, Prognostic and predictive values of long non-coding RNA LINC00472 in breast cancer, Oncotarget 6(11) (2015) 8579-92.

[11] N. Cheng, X. Li, C. Zhao, S. Ren, X. Chen, W. Cai, M. Zhao, Y. Zhang, J. Li, Q. Wang, C. Zhou, Microarray expression profile of long non-coding RNAs in EGFR-TKIs resistance of human non-small cell lung cancer, Oncology reports 33(2) (2015) 833-9.

[12] W. Song, Y.Y. Liu, J.J. Peng, H.H. Liang, H.Y. Chen, J.H. Chen, W.L. He, J.B. Xu, S.R. Cai, Y.L. He, Identification of differentially expressed signatures of long non-coding RNAs associated with different metastatic potentials in gastric cancer, Journal of gastroenterology 51(2) (2016) 119-29.

[13] J. Wang, Y.X. Song, Z.N. Wang, Non-coding RNAs in gastric cancer, Gene 560(1) (2015) 1-8.

[14] T. Arita, D. Ichikawa, H. Konishi, S. Komatsu, A. Shiozaki, K. Shoda, T. Kawaguchi, S. Hirajima, H. Nagata, T. Kubota, H. Fujiwara, K. Okamoto, E. Otsuji, Circulating long non-coding RNAs in plasma of patients with gastric cancer, Anticancer research 33(8) (2013) 3185-93.

[15] Q. Pang, J. Ge, Y. Shao, W. Sun, H. Song, T. Xia, B. Xiao, J. Guo, Increased expression of long intergenic non-coding RNA LINC00152 in gastric cancer and its clinical significance, Tumour biology : the journal of the International Society for Oncodevelopmental Biology and Medicine 35(6) (2014) 5441-7.

[16] H. Song, W. Sun, G. Ye, X. Ding, Z. Liu, S. Zhang, T. Xia, B. Xiao, Y. Xi, J. Guo, Long non-coding RNA expression profile in human gastric cancer and its clinical significances, Journal of translational medicine 11 (2013) 225.

[17] Bosman FT, Carneiro F, Hruban RH, et al.WHO Classification of Tumours, Fourth Edition. International Agency for Research on Cancer. France,2010,54.

[18] L. Dong, P. Qi, M.D. Xu, S.J. Ni, D. Huang, Q.H. Xu, W.W. Weng, C. Tan, W.Q. Sheng, X.Y. Zhou, X. Du, Circulating CUDR, LSINCT-5 and PTENP1 long noncoding RNAs in sera distinguish patients with gastric cancer from healthy controls, International journal of cancer 137(5) (2015) 1128-35.

[19] Y.S. Tong, X.W. Wang, X.L. Zhou, Z.H. Liu, T.X. Yang, W.H. Shi, H.W. Xie, J. Lv, Q.Q. Wu, X.F. Cao, 
Identification of the long non-coding RNA POU3F3 in plasma as a novel biomarker for diagnosis of esophageal squamous cell carcinoma, Molecular cancer 14 (2015) 3.

[20] C.L. Andersen, J.L. Jensen, T.F. Orntoft, Normalization of real-time quantitative reverse transcription-PCR data: a model-based variance estimation approach to identify genes suited for normalization, applied to bladder and colon cancer data sets, Cancer research 64(15) (2004) 5245-50.

[21] Q. Li, Y. Shao, X. Zhang, T. Zheng, M. Miao, L. Qin, B. Wang, G. Ye, B. Xiao, J. Guo, Plasma long noncoding RNA protected by exosomes as a potential stable biomarker for gastric cancer, Tumour biology : the journal of the International Society for Oncodevelopmental Biology and Medicine 36(3) (2015) 2007-12.

[22] X. Zhou, C. Yin, Y. Dang, F. Ye, G. Zhang, Identification of the long non-coding RNA H19 in plasma as a novel biomarker for diagnosis of gastric cancer, Scientific reports 5 (2015) 11516.

[23] A.N. Kallen, X.B. Zhou, J. Xu, C. Qiao, J. Ma, L. Yan, L. Lu, C. Liu, J.S. Yi, H. Zhang, W. Min, A.M. Bennett, R.I. Gregory, Y. Ding, Y. Huang, The imprinted H19 IncRNA antagonizes let-7 microRNAs, Molecular cell 52(1) (2013) 101-12.

[24] J. Zhou, L. Yang, T. Zhong, M. Mueller, Y. Men, N. Zhang, J. Xie, K. Giang, H. Chung, X. Sun, L. Lu, G.G. Carmichael, H.S. Taylor, Y. Huang, H19 IncRNA alters DNA methylation genome wide by regulating S-adenosylhomocysteine hydrolase, Nature communications 6 (2015) 10221.

[25] J.S. Chen, Y.F. Wang, X.Q. Zhang, J.M. Lv, Y. Li, X.X. Liu, T.P. Xu, H19 serves as a diagnostic biomarker and up-regulation of $\mathrm{H} 19$ expression contributes to poor prognosis in patients with gastric cancer, Neoplasma 63(2) (2016) 223-30.

[26] F. Yang, J. Bi, X. Xue, L. Zheng, K. Zhi, J. Hua, G. Fang, Up-regulated long non-coding RNA H19 contributes to proliferation of gastric cancer cells, The FEBS journal 279(17) (2012) 3159-65.

[27] H. Li, B. Yu, J. Li, L. Su, M. Yan, Z. Zhu, B. Liu, Overexpression of IncRNA H19 enhances carcinogenesis and metastasis of gastric cancer, Oncotarget 5(8) (2014) 2318-29.

[28] E.B. Zhang, L. Han, D.D. Yin, R. Kong, W. De, J. Chen, C-Myc-induced, long, noncoding H19 affects cell proliferation and predicts a poor prognosis in patients with gastric cancer, Medical oncology 31(5) (2014) 914.

[29] J. Ji, J. Tang, L. Deng, Y. Xie, R. Jiang, G. Li, B. Sun, LINC00152 promotes proliferation in hepatocellular carcinoma by targeting EPCAM via the mTOR signaling pathway, Oncotarget 6(40) (2015) 42813-24.

[30] J. Zhou, X. Zhi, L. Wang, W. Wang, Z. Li, J. Tang, J. Wang, Q. Zhang, Z. Xu, Linc00152 promotes proliferation in gastric cancer through the EGFR-dependent pathway, Journal of experimental \& clinical cancer research : CR 34 (2015) 135.

[31] J. Zhao, Y. Liu, W. Zhang, Z. Zhou, J. Wu, P. Cui, Y. Zhang, G. Huang, Long non-coding RNA Linc00152 is involved in cell cycle arrest, apoptosis, epithelial to mesenchymal transition, cell migration and invasion in gastric cancer, Cell cycle 14(19) (2015) 3112-23.

[32] W.M. Chen, M.D. Huang, D.P. Sun, R. Kong, T.P. Xu, R. Xia, E.B. Zhang, Y.Q. Shu, Long intergenic non-coding RNA 00152 promotes tumor cell cycle progression by binding to EZH2 and repressing p15 and p21 in gastric cancer, Oncotarget 7(9) (2016) 9773-87.

[33] D.B. Polk, R.M. Peek, Jr., Helicobacter pylori: gastric cancer and beyond, Nature reviews. Cancer 10(6) (2010) 403-14.

[34] R.M. Peek, Jr., M.J. Blaser, Helicobacter pylori and gastrointestinal tract adenocarcinomas, Nature reviews. Cancer 2(1) (2002) 28-37.

[35] H.M. Zeng, K.F. Pan, Y. Zhang, L. Zhang, J.L. Ma, T. Zhou, H.J. Su, W.Q. Li, J.Y. Li, M. Gerhard, M. 
Classen, W.C. You, Genetic variants of toll-like receptor 2 and 5, helicobacter pylori infection, and risk of gastric cancer and its precursors in a chinese population, Cancer epidemiology, biomarkers \& prevention : a publication of the American Association for Cancer Research, cosponsored by the American Society of Preventive Oncology 20(12) (2011) 2594-602.

[36] K.F. Pan, L. Formichella, L. Zhang, Y. Zhang, J.L. Ma, Z.X. Li, C. Liu, Y.M. Wang, G. Goettner, K. Ulm, M. Classen, W.C. You, M. Gerhard, Helicobacter pylori antibody responses and evolution of precancerous gastric lesions in a Chinese population, International journal of cancer 134(9) (2014) 2118-25.

[37] H. Zhu, Q. Wang, Y. Yao, J. Fang, F. Sun, Y. Ni, Y. Shen, H. Wang, S. Shao, Microarray analysis of Long non-coding RNA expression profiles in human gastric cells and tissues with Helicobacter pylori Infection, BMC medical genomics 8 (2015) 84.

[38] Y. Yao, Q. Jiang, L. Jiang, J. Wu, Q. Zhang, J. Wang, H. Feng, P. Zang, Lnc-SGK1 induced by Helicobacter pylori infection and highsalt diet promote Th2 and Th17 differentiation in human gastric cancer by SGK1/Jun B signaling, Oncotarget (2016).

[39] L. Lu, H.A. Risch, Exosomes: potential for early detection in pancreatic cancer, Future oncology 12(8) (2016) 1081-90.

[40] W.K. Leung, M.S. Wu, Y. Kakugawa, J.J. Kim, K.G. Yeoh, K.L. Goh, K.C. Wu, D.C. Wu, J. Sollano, U. Kachintorn, T. Gotoda, J.T. Lin, W.C. You, E.K. Ng, J.J. Sung, C. Asia Pacific Working Group on Gastric, Screening for gastric cancer in Asia: current evidence and practice, The Lancet. Oncology 9(3) (2008) 279-87. 
Table 1. Basic characteristics of the study subjects.

\begin{tabular}{|c|c|c|c|}
\hline Characteristics & $\begin{array}{l}\text { GC Patients } \\
(n=133)\end{array}$ & $\begin{array}{l}\text { Controls } \\
(n=152)\end{array}$ & $P$ \\
\hline \multicolumn{4}{|l|}{ Age, years } \\
\hline$<60$ & $59(44.4)$ & $74(48.7)$ & 0.465 \\
\hline$\geq 60$ & $74(55.6)$ & $78(51.3)$ & \\
\hline \multicolumn{4}{|l|}{ Gender } \\
\hline Male & $103(77.4)$ & $116(76.3)$ & 0.822 \\
\hline Female & $30(22.6)$ & $36(23.7)$ & \\
\hline \multicolumn{4}{|l|}{ Smoking } \\
\hline Ever or current & $63(47.4)$ & $68(44.7)$ & 0.492 \\
\hline Never & $66(49.6)$ & $84(55.3)$ & \\
\hline Missing & $4(3.0)$ & $0(0.0)$ & \\
\hline \multicolumn{4}{|l|}{ Drinking } \\
\hline Ever or current & $56(42.1)$ & $35(23.0)$ & $<0.001$ \\
\hline Never & $73(54.9)$ & $117(77.0)$ & \\
\hline Missing & $4(3.0)$ & $0(0.0)$ & \\
\hline \multicolumn{4}{|l|}{ H. pylori infection } \\
\hline Positive & $123(92.5)$ & $91(59.9)$ & $<0.001$ \\
\hline Negative & $10(7.5)$ & $61(40.1)$ & \\
\hline \multicolumn{4}{|c|}{ Type of $H$. pylori infection } \\
\hline $\mathrm{CagA}+$ & $90(67.6)$ & $50(32.9)$ & $<0.001$ \\
\hline VacA+ & $69(51.8)$ & $36(23.7)$ & $<0.001$ \\
\hline Type I & $91(68.4)$ & $57(37.5)$ & $<0.001$ \\
\hline Type II & $32(24.1)$ & $34(22.4)$ & 0.736 \\
\hline \multicolumn{4}{|l|}{ Stage } \\
\hline Early & $40(30.1)$ & & \\
\hline Advanced & $57(42.8)$ & & \\
\hline Missing & $36(27.1)$ & & \\
\hline \multicolumn{4}{|l|}{ Location } \\
\hline Cardia & $30(22.6)$ & & \\
\hline Non-cardia & $100(75.2)$ & & \\
\hline Missing & $3(2.2)$ & & \\
\hline
\end{tabular}


Table 2. H19 and LINC00152 expression status in GC cases and controls.

\begin{tabular}{lllll}
\hline LncRNA levels & Control, n(\%) & GC, n(\%) & OR $(95 \%$ CI $)$ & $P$ \\
\hline H19 & & & & \\
Low & $76(50.0)$ & $43(32.3)$ & 1 & \\
High & $76(50.0)$ & $90(67.7)$ & $2.17(1.21-3.88)$ & $0.009^{\mathrm{a}}$ \\
LINC00152 & & & & \\
Low & & & & \\
High & $76(50.0)$ & $57(42.9)$ & 1 & \\
Combined H19 and LINC00152 levels & & $76(57.1)$ & $2.09(1.18-3.70)$ & $0.012^{\mathrm{b}}$ \\
Both low expression & $76(50.0)$ & & & \\
Only H19 high expression & & & & \\
Only LINC00152 high expression & $51(33.6)$ & $32(24.0)$ & $1.56(0.63-3.86)$ & $0.338^{\mathrm{c}}$ \\
Both high expression & $51(33.6)$ & $46(34.6)$ & $1.69(0.70-4.12)$ & $0.275^{\mathrm{c}}$ \\
\hline
\end{tabular}

${ }^{\mathrm{a}}$ Unconditional logistic regression adjusted for age, gender, $H$. pylori infection, smoking, drinking and LINC00152 levels.

${ }^{\mathrm{b}}$ Unconditional logistic regression adjusted for age, gender, $H$. pylori infection, smoking, drinking and H19 levels.

${ }^{c}$ Unconditional logistic regression adjusted for age, gender, H. pylori infection, smoking and drinking. 
Table 3. Stratified analysis on the association of serum lncRNAs expression levels with the risk of GC by $H$. pylori infection status.

\begin{tabular}{|c|c|c|c|c|c|c|}
\hline lncRNA & $\begin{array}{l}\text { H. pylori } \\
\text { infection }\end{array}$ & $\begin{array}{l}\text { Expression } \\
\text { level }\end{array}$ & $\begin{array}{l}\text { Control, } \\
\mathrm{n}(\%)\end{array}$ & $\mathrm{GC}, \mathrm{n}(\%)$ & OR $(95 \% \mathrm{CI})$ & $P$ \\
\hline \multirow[t]{4}{*}{ H19 } & Negative & Low & $32(52.5)$ & $5(50.0)$ & 1.00 & \\
\hline & Negative & High & $29(47.5)$ & $5(50.0)$ & $1.21(0.29-5.02)$ & $0.793^{\mathrm{a}}$ \\
\hline & Positive & Low & $44(48.4)$ & $38(30.9)$ & 1.00 & \\
\hline & Positive & High & $47(51.6)$ & $85(69.1)$ & $2.27(1.19-4.33)$ & $0.013^{\mathrm{a}}$ \\
\hline \multirow[t]{4}{*}{ LINC00152 } & Negative & Low & $26(42.6)$ & $3(30.0)$ & 1.00 & \\
\hline & Negative & High & $35(57.4)$ & $7(70.0)$ & $2.25(0.49-10.27)$ & $0.297^{\mathrm{b}}$ \\
\hline & Positive & Low & $50(54.9)$ & $54(43.9)$ & 1.00 & \\
\hline & Positive & High & $41(45.1)$ & $69(56.1)$ & $2.04(1.09-3.82)$ & $0.026^{\mathrm{b}}$ \\
\hline
\end{tabular}

${ }^{\mathrm{a}}$ Unconditional logistic regression adjusted for age, gender, smoking, drinking and

LINC00152 levels.

${ }^{\mathrm{b}}$ Unconditional logistic regression adjusted for age, gender, smoking, drinking and H19 levels. 
Table 4. Analysis of joint effects between lncRNAs expression and $H$. pylori infection on risk of GC.

\begin{tabular}{clllll}
\hline LncRNA levels & H. pylori infection & Control, $(\%)$ & $\mathrm{GC}, \mathrm{n}(\%)$ & OR $(95 \% \mathrm{CI})$ & $P$ \\
\hline H19 & & & & & \\
Low & Negative & $32(21.1)$ & $5(3.8)$ & 1 & \\
Low & Positive & $44(28.9)$ & $38(28.5)$ & $5.75(1.97-16.66)$ & $0.001^{\mathrm{a}}$ \\
High & Negative & $29(19.1)$ & $5(3.8)$ & $1.36(0.35-5.33)$ & $0.662^{\mathrm{a}}$ \\
High & Positive & $47(30.9)$ & $85(63.9)$ & $13.75(4.75-39.84)$ & $<0.001^{\mathrm{a}}$ \\
LINC00152 & & & & \\
Low & Negative & $26(17.1)$ & $3(2.2)$ & 1 & \\
Low & Positive & $50(32.9)$ & $54(40.6)$ & $8.55(2.38-30.78)$ & $0.001^{\mathrm{b}}$ \\
High & Negative & $35(23.0)$ & $7(5.3)$ & $2.36(0.54-10.34)$ & $0.255^{\mathrm{b}}$ \\
High & Positive & $41(27.0)$ & $69(51.9)$ & $17.49(4.78-63.92)$ & $<0.001^{\mathrm{b}}$ \\
\hline
\end{tabular}

${ }^{\mathrm{a}}$ Unconditional logistic regression adjusted for age, gender, smoking, drinking and LINC00152 levels.

${ }^{\mathrm{b}}$ Unconditional logistic regression adjusted for age, gender, smoking, drinking and H19 levels. 
Table 5. Stratified analysis on the association between serum lncRNAs expression levels with the risk of GC by type of $H$. pylori infection

\begin{tabular}{|c|c|c|c|c|c|}
\hline \multirow{2}{*}{$\begin{array}{l}\text { LncRNA } \\
\text { expression status }\end{array}$} & \multirow{2}{*}{$\begin{array}{l}\text { Type of } \\
\text { H. pylori infection }\end{array}$} & \multirow{2}{*}{$\begin{array}{l}\text { Control, } \mathrm{n}(\%) \\
\mathrm{N}=91\end{array}$} & \multirow{2}{*}{$\begin{array}{l}\mathrm{GC}, \mathrm{n}(\%) \\
\mathrm{N}=123\end{array}$} & \multirow{2}{*}{ OR $(95 \% \mathrm{CI})^{\mathrm{a}}$} & \multirow{2}{*}{$P$} \\
\hline & & & & & \\
\hline \multicolumn{6}{|l|}{ H19 } \\
\hline Low & Type I & $32(56.1)$ & $27(29.7)$ & 1.00 & \\
\hline High & Type I & $25(43.9)$ & $64(70.3)$ & $4.23(1.80-9.94)$ & 0.001 \\
\hline Low & Type II & $12(35.3)$ & $11(34.4)$ & 1.00 & \\
\hline High & Type II & $22(64.7)$ & $21(65.6)$ & $0.76(0.24-2.48)$ & 0.654 \\
\hline \multicolumn{6}{|l|}{ LINC00152 } \\
\hline Low & Type I & $33(57.9)$ & $41(45.1)$ & 1.00 & \\
\hline High & Type I & $24(42.1)$ & $50(54.9)$ & $2.86(1.22-6.69)$ & 0.015 \\
\hline Low & Type II & $17(50.0)$ & $13(40.6)$ & 1.00 & \\
\hline High & Type II & $17(50.0)$ & $19(59.4)$ & $1.59(0.54-4.68)$ & 0.401 \\
\hline
\end{tabular}

${ }^{\mathrm{a}}$ Unconditional logistic regression adjusted for age, gender, smoking and drinking.

\section{Figure Legends}

Fig. 1. Comparison of serum lncRNAs between pre- and postoperative samples from GC patients. (a): Box plots of pre and postoperative H19 expression levels. The levels were significantly reduced in postoperative plasma samples $(P=0.010)$. The upper and lower limits of the boxes and the lines inside the boxes indicate the $75^{\text {th }}$ and $25^{\text {th }}$ percentiles and the median, respectively. (b): Box plots of pre and postoperative LINC00152 expression levels. There were no significant difference in the level of LINC00152 between pre- and postoperative samples from GC patients $(P=0.520)$. 
Fig. 2. The diagnostic models of H19, LINC00152 and H. pylori infection status. Receiver operating characteristic (ROC) curves showed diagnostic sensitivity and specificity of each panel. 


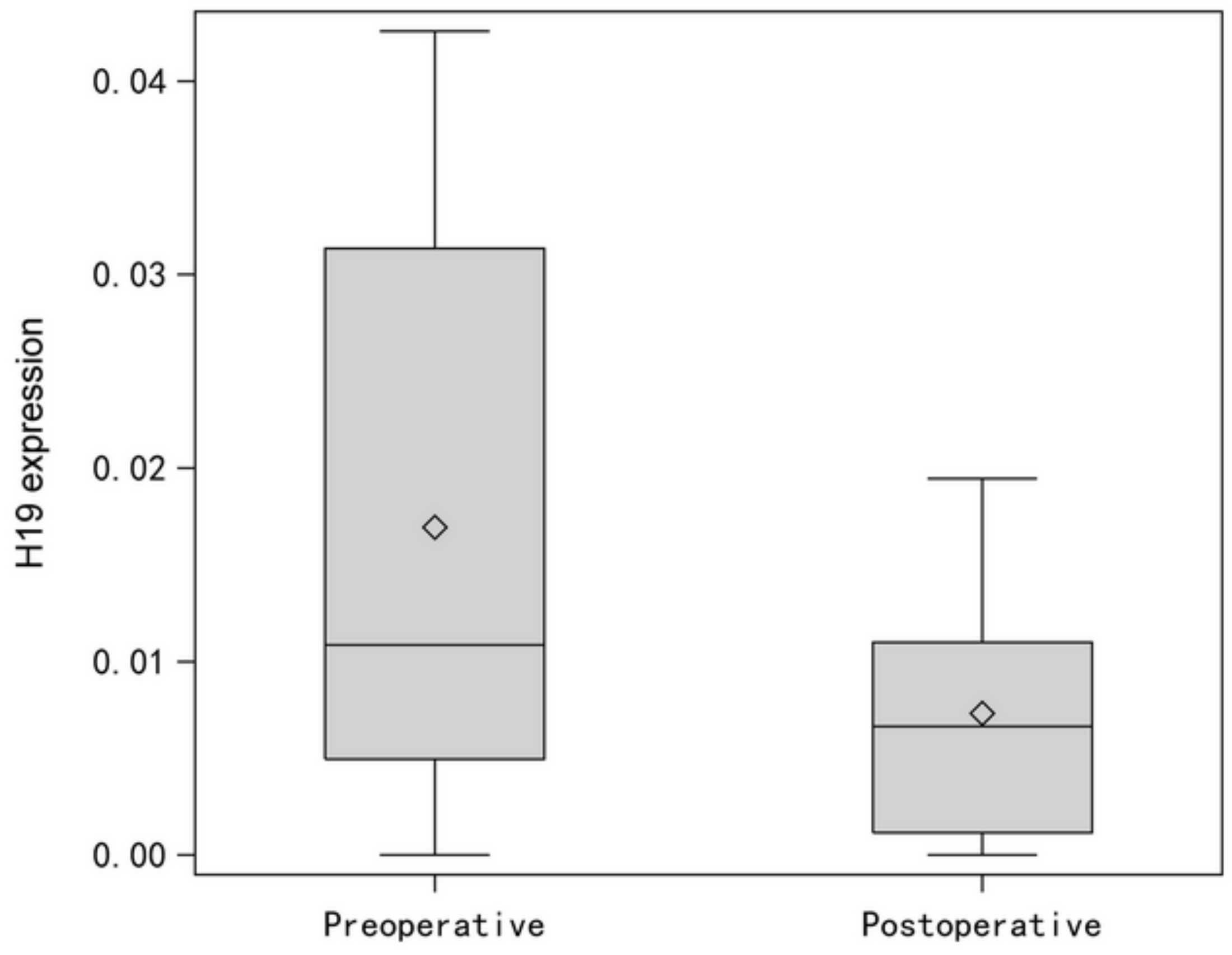

$1 a$ 


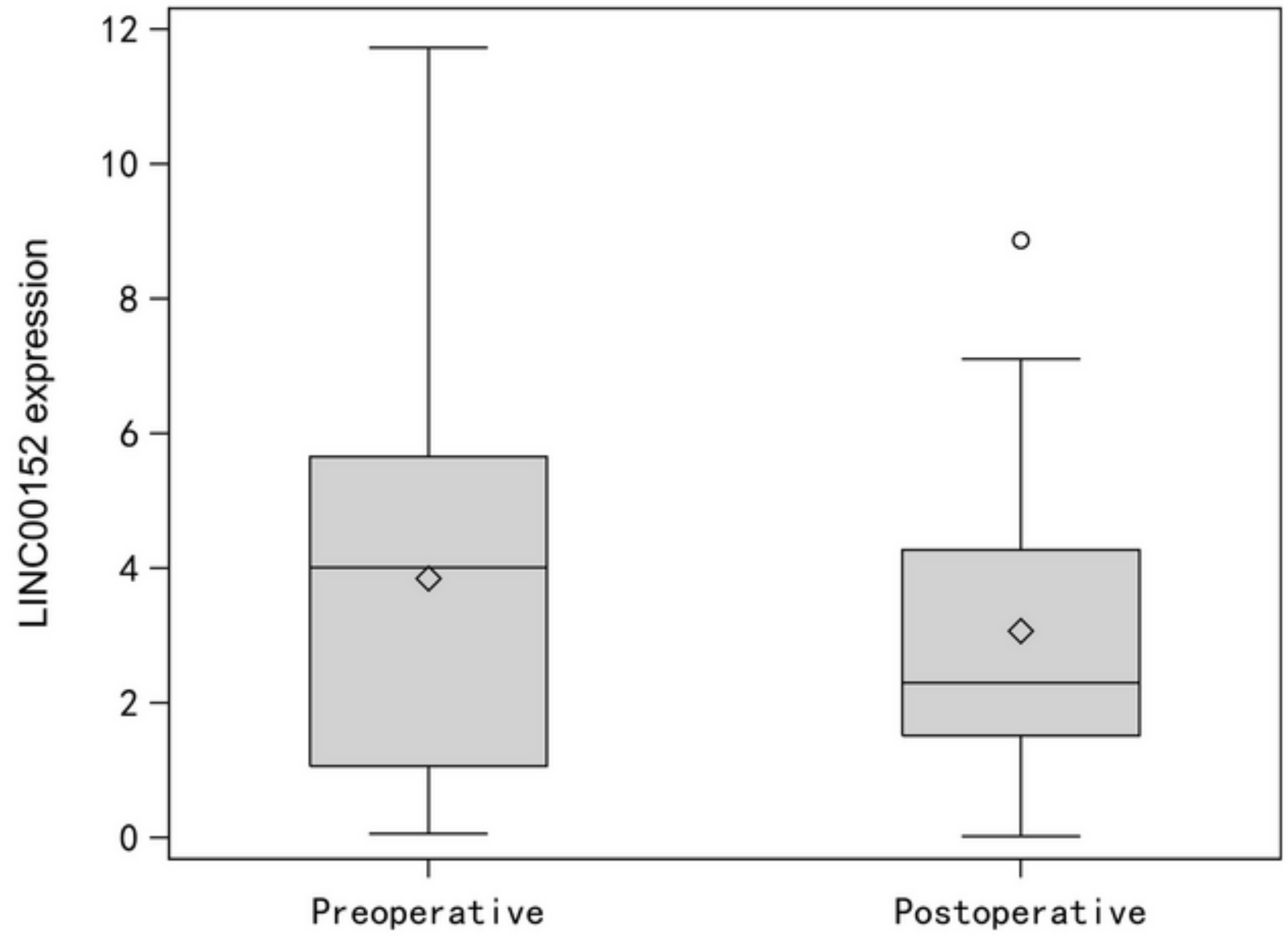

$1 b$ 


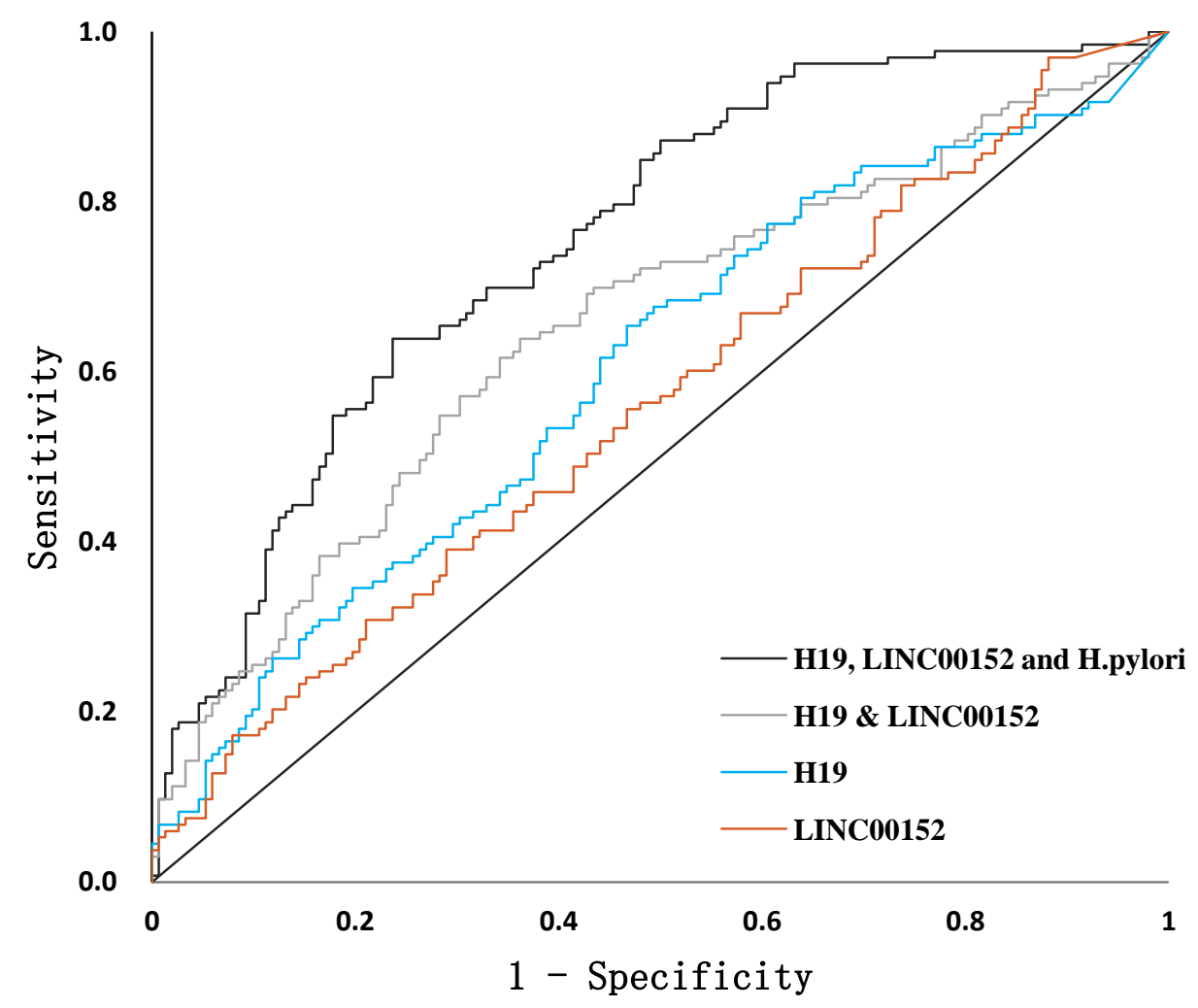

Fig. 2. The diagnostic models of H19, LINC00152 and H. pylori infection status. Receiver operating characteristic (ROC) curves showed diagnostic sensitivity and specificity of each panel. 